\title{
Active crowd sensing
}

\author{
Zhiyong $\mathrm{Yu}^{1}$ - Jiangtao Wang ${ }^{2}$. Jordán Pascual Espada ${ }^{3}$
}

(C) The Author(s), under exclusive licence to Springer-Verlag London Ltd., part of Springer Nature 2021

\section{Introduction}

Mobile Crowd Sensing (MCS) has emerged as a paradigm for gathering information about the physical world. Using smartphones, networked vehicles and other sensor-rich mobile, portable and wearable devices, massive volumes of data can be gathered, converged and mined to enable applications in intelligent traffic, environmental monitoring, urban planning or public safety management, amongst many others.

The cost of crowdsensed data and the quality of data are two key factors for MCS. There has been a great deal of work to optimize them separately such as recruiting participants that can meet a task completion requirement, or utilizing data already collected to infer uncollected data with minimal error. However, it is a promising direction to optimize both cost and quality jointly. This idea is originated from active learning, which selects the most useful samples and so reduces the number of labeled samples needed. This idea has been successfully used in other research fields including signal processing and image processing.

In MCS, this is known as "Active Crowd Sensing", and presents several challenges including: the crowdsensed data are spatiotemporally autocorrelated, while traditional active learning assumes the data are independently identically distributed; and that data from different applications require

\section{Zhiyong $\mathrm{Yu}$}

yuzhiyong@fzu.edu.cn

Jiangtao Wang

jiangtao.wang@coventry.ac.uk

Jordán Pascual Espada

pascualjordan@uniovi.es

1 College of Mathematics and Computer Science, Fuzhou University, Fuzhou, China

2 Center for Intelligent Healthcare, Coventry University, Coventry, UK

3 Department of Computer Science, University of Oviedo, Oviedo, Spain different learning algorithms and so active selection strategies should be based on specific learning algorithms.

This special issue promoted some researches addressing the above challenges in the form of original problem modeling and framework developing, new datasets/features that can be easily accessed and helpful for label inferring, and novel applications and systems in Active Crowd Sensing. We first summarize in Section 2 these 10 articles that have been accepted, and then discuss in Section 3 the conclusion and further topics.

\section{Accepted articles}

A general MCS system framework usually consists of four phases: participant selection, sensing, data transmission, and data utilizing. Each phase has one or more articles to discuss the corresponding topics.

In the participant selection phase, Tao et al. [1] proposed a novel quality-aware participant reputation model with active factors, and two kinds of sensing-gain constrained participant selection mechanisms with both sufficient and insufficient user resources, i.e., Multi-Stage Decision mechanism via Greedy strategy (MSD-G), and Sensing-Gain Constrained Dynamic Programming (SGC-DP), respectively.

In the sensing phase, Dai et al. [2] designed a lightweight augmented reality system, called LAR, which can recognize the target object quickly and precisely by combining the distance with image feature and then matches with those images stored in database. Cheng et al. [3] proposed a visual saliency model based on gaze recall with self-reporting from crowd workers. They conducted optimizations for the parameters in the data acquisition stage so that the accuracy of the crowdsourcing eye tracking data reached one degree of visual angle.

In the data transmission phase, $\mathrm{He}$ et al. [4] provided a spatiotemporal opportunistic transmission method which searches publishers or participants of sensing tasks in a space-time unit according to the spatiotemporal encountering probabilities, and tracks the publishers or participants across the space-time units according to the spatiotemporal visiting 
probabilities. Ren et al. [5] proposed a Data Relay Mule-based Collection Scheme (DRMCS) to improve the sensing task completion rate, redundancy rate and delay. In the scheme, the Micro Mobile Data Centers are designed and later selected to connect the huge number of intelligent sensing devices. By using reinforcement learning, Zhao et al. [6] derived an adaptive transmission policy for underground sensors to efficiently use their energy and avoid transmitting sensory data in unreliable paths under a dynamic environment.

In the data utilizing phase, Shi et al. [7] introduced a data recovery algorithm based on generative adversarial networks. The convolution neural network is used as the basic model of this algorithm. Zhao et al. [8] tackled the uncertainty of taxi demands and the impact of the parallel car-hailing markets (e.g., Uber demands) on taxi demands through a unified framework that can use multi-source data systematically. Gao et al. [9] conducted sparse representation on taxi activity over spatially partitioned cells in a city. They can perceive the deviation from the normal evolution of traffic flows and find the traffic anomalies. Zhou et al. [10] conducted a spatiotemporal study to understand urban crimes leveraging multisource crowd sensed data, including crime data, meteorological data, POI distribution, and taxi trips.

\section{Conclusion}

Although this special issue has covered a number of topics of Active Crowd Sensing, such as optimization both or tradeoff between the cost and the quality of crowdsensed data, some other but also interesting topics need to be further explored, including: sparse mobile crowdsensing, relations between sensing rate and learning rate, sub-sampling and superresolution for crowdsensed data, active deep/transfer learning in MCS, etc.

\section{References}

1. Tao D, Gao R, Sun H (2021) Sensing-gain constrained participant selection mechanism for Mobile Crowdsensing. Personal Ubiquitous Comput. https://doi.org/10.1007/s00779-020-01470-8

2. Dai X, Shang F, Xing T, Chen F, Liu B (2021) LAR: a low power, high-precision Mobile phone-based AR system. Personal Ubiquitous Comput. https://doi.org/10.1007/s00779-020-01421-3

3. Cheng S, Fan J, Hu Y (2021) Visual saliency model based on crowdsourcing eye tracking data and its application in visual design. Personal Ubiquitous Comput. https://doi.org/10.1007/ s00779-020-01463-7

4. He X, Liu M, Yang G. (2021) Spatiotemporal opportunistic transmission for Mobile crowd sensing networks. Personal Ubiquitous Comput. https://doi.org/10.1007/s00779-020-01439-7

5. Ren Y, Wang T, Zhang S, Zhang J (2021) An intelligent big data collection technology based on micro Mobile data centers for Crowdsensing vehicular sensor network. Personal Ubiquitous Comput. https://doi.org/10.1007/s00779-020-01440-0

6. Zhao D, Zhou Z, Wang S, Liu B, Gaaloul W (2021) Reinforcement learning-enabled efficient data gathering in underground wireless sensor networks. Personal Ubiquitous Comput. https://doi.org/10. 1007/s00779-020-01443-x

7. Shi Y, Zhang X, Hu Q, Cheng H (2021) Data recovery algorithm based on generative adversarial networks in crowd sensing internet of things. Personal Ubiquitous Comput. https://doi.org/10.1007/ s00779-020-01428-w

8. Zhao J, Chen C, Huang H, Xiang C (2021) Unifying Uber and taxi data via deep models for taxi-passenger demand prediction. Personal Ubiquitous Comput. https://doi.org/10.1007/s00779-02001426-y

9. Gao J, Zheng D, Yang S (2021) Perceiving spatiotemporal traffic anomalies from sparse representation-Modeled City dynamics. Personal Ubiquitous Comput. https://doi.org/10.1007/s00779-02001474-4

10. Zhou B, Chen L, Zhao S, Li S, Pan G (2021) Spatio-temporal analysis of urban crime leveraging multisource Crowdsensed data. Personal Ubiquitous Comput. https://doi.org/10.1007/s00779-02001456-6

Publisher's note Springer Nature remains neutral with regard to jurisdictional claims in published maps and institutional affiliations. 